\title{
Compreensão da morte e desenvolvimento Humano: contribuições à Psicologia Hospitalar
}

\section{Understanding Death and Human Development: contributions to Hospital Psychology}

\section{Jean Von Hohendorff}

Acadêmico do curso de Psicologia da FACCAT - Faculdades Integradas de Taquara, Taquara, RS, Brasil

\section{Wilson Vieira de Melo *}

Diretor e professor do curso de Especialização em Psicoterapia CognitivoComportamental da WP - Centro de Psicoterapia Cognitivo-Comportamental, Porto Alegre, RS, Brasil

Professor do curso de Psicologia da FACCAT - Faculdades Integradas de Taquara, Taquara, RS, Brasil

\begin{abstract}
Resumo
Este estudo apresenta uma revisão teórica sobre a compreensão da morte nas diferentes etapas do desenvolvimento humano com o objetivo de contribuir para o trabalho de psicólogos hospitalares. Foram realizadas consultas a artigos indexados na língua portuguesa, além de livros de autores importantes. Foi possível observar que a morte é compreendida de maneira distinta durante as fases do desenvolvimento humano, sofrendo influência de alguns fatores, tais como: cultura, informações e experiências com a situação. Os autores propõem que a compreensão da morte pode ser vista em um continuum. Além disso, um quadro no qual foram incluídos os principais aspectos da compreensão da morte é proposto com vistas a facilitar o entendimento dos resultados deste estudo.
\end{abstract}

Palavras-chave: Morte, Desenvolvimento humano, Psicologia hospitalar.

\begin{abstract}
This study presents a theoretical revision of the way in which death is understood at different stages of the cycle of life, with view to contribute to hospital psychology work. In order to construct this research, articles in the Portuguese language were consulted as well as other works by eminent authors. From this study it was possible to observe that death is understood in a distinct way during the phases of life, being influenced by factors such as culture, information and experiences of the situation. The authors propose that the understanding of death can be seen in a continuum. Furthermore, a table in which were included the main aspects of death understanding is presented to facilitate the comprehension of the results of this study.
\end{abstract}

Keywords: Death, Human development, Hospital psychology. 


\section{I ntrodução}

O que pode haver de interessante em investigar um tema como a morte? Atualmente nos deparamos com uma negação acerca desse tema (SILVA, 2003). A sociedade, marcada por um ritmo alucinante, parece ter deixado de lado o fato de todos nós sermos seres finitos. Assim, o homem tende a não pensar sobre sua finitude e a das pessoas que o rodeiam. Nota-se um despreparo no que diz respeito ao enfrentamento dessa situação. Além da população em geral, esse despreparo está presente em profissionais ligados à saúde, que têm sua formação voltada para a vida (FIDELIS, 2001).

Sendo a psicologia uma ciência que lida com seres humanos e suas vidas e, conseqüentemente, com a morte, tal assunto torna-se relevante. Especialmente, dentro da psicologia hospitalar, é necessário estar preparado para situações de finitude (KOVÁCS, 1989). O hospital é o lugar onde as pessoas buscam ajuda para restituir a saúde. Entretanto, a vida também pode chegar ao seu fim neste local. Atualmente, é cada vez mais freqüente que a morte venha a ocorrer em hospitais, devido, principalmente, aos avanços da medicina (OKAMOTO, 2004). Sendo assim, é imprescindível que o psicólogo que atua neste contexto esteja preparado.

A maneira como a morte é compreendida é dinâmica ao longo do desenvolvimento humano. Desde a infância, as pessoas têm contato com perdas, mas é a partir da adolescência que realmente entendemos o significado da morte. $\mathrm{Na}$ idade adulta evidenciamos tal fato como algo possível de acontecer, mas é na velhice que sua possibilidade parece ser mais aceita, uma vez que tal etapa é encarada como última no ciclo de desenvolvimento humano. Além das variáveis relacionadas com o desenvolvimento humano, a cultura e as situações de perda que vivenciamos contribuem para que formemos nossa visão sobre a finitude humana.

Para um melhor entendimento acerca do assunto, faz-se importante o estudo sobre a temática. Sendo assim, o presente trabalho tem como objetivo realizar uma revisão teórica de estudos que abordaram a temática da compreensão da morte ao longo do desenvolvimento humano. Com isso, pretende-se aprofundar o conhecimento sobre o tema, através de reflexões acerca do material coletado.

Para que o objetivo deste trabalho fosse alcançado, foram realizadas consultas a artigos indexados publicados na língua portuguesa nos bancos de dados Scielo, BVS-Psi e CNPQ, além de alguns artigos importantes citados nessas publicações. Os descritores utilizados foram "morte", "luto", "infância", "adolescência", "idade adulta", "terceira idade", "velhice", "idoso", "psicologia hospitalar". Os trabalhos que não estavam 
disponíveis na íntegra foram utilizados apenas para consulta do resumo. Ademais, foram pesquisados livros de autores importantes da construção da teoria (ALMEIDA, 2000; ASSUMPÇÃO, 2001; BEE, 1997; BROWN, 2001; ELKIND, 1984; KOVÁCS, 1992; KÜBLER-ROSS, 2005; PAPALIA; OLDS, 2000).

Após a leitura do material pesquisado, procurou-se construir um texto que abarcasse a compreensão da morte ao longo do desenvolvimento humano com vistas a contribuir para aqueles profissionais ligados a área de cuidados psicológicos, especialmente o psicólogo hospitalar. Para tal, foram considerados os principais resultados dos estudos investigados.

\section{Considerações sobre a psicologia hospitalar e sua interface com a finitude humana}

Almeida (2000) evidencia que o psicólogo hospitalar tem papel na humanização dos cuidados com o paciente; no atendimento ao paciente e sua família; na elaboração da experiência vivida com a hospitalização, além de atuar junto à equipe de saúde. Além disso, o profissional presente em hospitais terá que lidar com a morte, pois sendo um profissional de saúde, tal situação fará parte de seu cotidiano profissional (KOVÁCS, 1989). No que diz respeito ao estudo da morte (tanatologia), Wilma da Costa Torres foi pioneira. Tal assunto, além de presente no seu cotidiano profissional, foi tema de estudos, tendo diversas publicações sobre o assunto (KOVÁCS, 2004). Evidencia-se assim, a importância e a relevância de se estudar tal assunto, especialmente no que tange aos profissionais que trabalham em hospitais.

Apesar de a morte ser reconhecida como natural, universal e inevitável, o homem é incapaz de imaginar a sua própria morte (COSTA, 1999) e, por isso, na sociedade, a maioria das pessoas tende a evitá-la (KÜBLERROSS, 2005). Tal situação, segundo Silva (2003), contribui para que ocorra um despreparado para lidar com a finitude humana.

A morte é um acontecimento que faz parte do desenvolvimento e está presente no cotidiano de todos (COMBINATO; QUEIROZ, 2005), porém está inserida em um contexto sócio histórico de negação (QUINTANA; ARPINI, 2002). Vários são os motivos para tal negação. Segundo Bellato e Carvalho (2005) através dos novos conhecimentos e técnicas adquiridos, a medicina busca ludibriar a morte, sendo considerado um fato natural somente na velhice. O modo como a nossa finitude foi e é encarada pelo homem ao longo do tempo é o tema de um estudo realizado por Araujo e Vieira (2001), no qual as autoras relatam que na Antigüidade, a morte era tratada com certo romantismo, embora os mortos fossem temidos e se procurava mantê-los afastados. Já na Idade Média, o homem convivia de forma mais harmoniosa com a morte, sem grandes temores e era permitido que as crianças participassem dos seus rituais. Por fim, nos 
tempos modernos, a morte passou a ser negada e a ser vista como representação de fracasso e interrupção nos projetos de vida.

Cada vez mais autores procuram mostrar que a morte pode ser encarada como um acontecimento que faz parte do ciclo vital de todos os seres (PAZIN-FILHO, 2005; TONETTO; RECH, 2001) e, assim, enfrentá-la de forma menos temerosa. Cabe aos profissionais de saúde a responsabilidade de discutir e refletir sobre este assunto para que possam oferecer subsídios a quem necessite (SOUZA; BOEMER, 2005). Para profissionais de saúde ou futuros profissionais o assunto abordado neste texto é uma constante, seja por situações vivenciadas ou pela iminência delas. Kovács (1989) lembra que a morte deve ser uma preocupação para a psicologia, embora possa ser evitada em nossa sociedade, a mesma negação não deveria ocorrer por parte do psicólogo.

Para o psicólogo hospitalar tal tema é ainda mais presente, fazendo-se necessário o conhecimento sobre o modo como as pessoas reagem perante a morte. Embora a profissionalização na área da saúde esteja voltada para uma ênfase na vida (FIDELIS, 2001), a morte sempre estará presente, necessitando preparo para o auxílio àqueles que a vivenciam. Assim, é fundamental que o profissional saiba como essa situação é compreendida durante as diferentes etapas da vida.

\section{A compreensão da morte ao longo do desenvolvimento humano}

O ser humano se diferencia por ser o único a ter consciência sobre sua finitude (MELO; VALLE, 1999), sendo que tal percepção se inicia na infância, definida como o período que se estende dos zero aos 12 anos de idade incompletos (PAPALIA; OLDS, 2000). Nunes, Carraro, Jou e Sperb (1998), realizaram estudo que teve como objetivo investigar como as crianças elaboram o conceito de morte. Partindo de uma perspectiva desenvolvimental cognitiva, foi resgatada a teoria de Jean Piaget para embasar a pesquisa. Os conceitos de irreversibilidade (alguém que morre não pode voltar a viver), não-funcionalidade (com a morte cessam as funções vitais) e universalidade (todos os seres-vivos morrem) são aspectos fundamentais para a obtenção do conceito de morte. Esses são adquiridos no estágio operatório concreto da teoria de Jean Piaget, no qual a criança passa a entender a reversibilidade das coisas que a cercam. Conhecendo a reversibilidade, a criança consegue conceber a irreversibilidade.

Em pesquisa realizada com crianças de dois a cinco anos vítimas de câncer, Almeida (2005) constatou que as crianças possuíam inabilidade para compreender a morte como sendo irreversível. Porém, no estudo de Nunes, Carraro, J ou e Sperb (1998), realizado com seis crianças entre seis e sete anos que foram investigadas através de entrevistas e desenhos, foi possível verificar que tais crianças mostraram compreender a 
irreversibilidade da morte. Cabe salientar que além da influência do desenvolvimento cognitivo, a experiência da criança com relação à morte e as representações formais à disposição na cultura também estão relacionadas com a elaboração do conceito de morte. Tais fatos podem explicar a diferença constatada entre os estudos.

Torres (2002) buscou investigar a aquisição do conceito de morte em crianças portadoras de diferentes patologias, comparando-as com crianças sadias de mesma condição sócio-econômica e mesmo nível cognitivo. Através do estudo, concluiu-se que há relação entre a aquisição do conceito de morte e o nível de desenvolvimento cognitivo da criança. Sendo assim, podemos perceber, dentre os outros fatores relacionados, a importância do desenvolvimento cognitivo para a compreensão da morte, fazendo-se necessária a adequação das informações sobre a morte para o entendimento infantil. Tal fato é citado por Gauderer (1987), pois muitas vezes os adultos tendem a dar-lhes respostas inadequadas, tais como "papai do céu levou" ou "foi fazer uma viagem muito longa" (ASSUMPÇÃO, 2001, p. 12).

Segundo Mazorra (2001), a morte de um genitor se caracteriza como uma das experiências de maior impacto sobre a criança, podendo desencadear quadros psicopatológicos na idade adulta. Em pesquisa realizada, Zavaschi et. al., (2002), foram encontrados vários estudos que evidenciaram a associação entre trauma na infância, tais como a perda de vínculos afetivos devido à morte de pais ou de irmãos, e depressão na vida adulta. Franco e Mazorra (2007) realizaram estudo com cinco crianças de três a oito anos investigando as fantasias dessas crianças e sua relação com o processo de elaboração e luto de seus genitores. 0 estudo obteve a conclusão de que o sentimento de desamparo predomina na mobilização de fantasias, uma vez que a morte de um ou ambos os pais gera sentimentos de ameaça à sobrevivência física e emocional da criança. Dentre as fantasias, estavam a de culpa pela morte do pai/mãe que tende a dificultar o processo de elaboração dessa perda. Finalmente, o estudo conclui que através das fantasias é possível conhecer o processo de elaboração do luto, além da compreensão de sentimentos, comportamentos e sintomas.

Após a infância, chegamos à adolescência, etapa na qual o jovem se depara com uma importante tarefa desenvolvimental, a construção de sua identidade. Nessa etapa da vida o jovem entende o significado da morte, porém, habitualmente não pensa muito sobre este fato. Rodriguez (2005), evidencia que os adolescentes possuem dificuldade para pensar na possibilidade de perder pessoas próximas, chegando a não perceber sua morte como possível, provavelmente pelos seus sentimentos de imortalidade e onipotência. Rodriguez e Kovács (2005) relatam que o jovem se encontra no auge da vida, buscando seu lugar no mundo e consolidando sua identidade, não sobrando espaço para pensar em sua 
finitude. Por outro lado, Günther (1996), buscando conhecer as preocupações de adolescentes entre 11 e 18 anos, verificou que $50 \%$ ou mais dos jovens se preocupava, entre outros, com a morte de algum familiar e com a possibilidade da perda de um amigo (a) próximo (a).

O fato de não pensarem muito sobre o assunto não impede que os adolescentes percebam as características essenciais da morte, estando somente afastados emocionalmente dela (KOVÁCS, 1992). Isso porque estão passando por diversas mudanças e sentimentos acreditando estar distante da mesma... (RODRIGUEZ; KOVÁCS, 2005). Outro fator que colabora para que os adolescentes em sua maioria não pensem no assunto morte é o fato de acreditarem que são invulneráveis. Elkind (1984) nomeia tal pensamento como fábula pessoal, ou seja, o pensamento que $o$ adolescente tem de não ser vulnerável aos acontecimentos vitais. Tal pensamento fica evidente no estudo realizado por Rodriguez e Kovács (2005), no qual as autoras nos dizem que comportamentos como o uso de drogas, direção arriscada levando a acidentes envolvendo adolescentes podem ser explicados pela necessidade de viver a vida intensamente e, assim, desafiar a morte.

Domingos e Maluf (2003) realizaram estudo com adolescentes, investigando as experiências de perda e luto nos mesmos. As reações iniciais (na ocasião da notícia do ocorrido) descritas foram: choque, descrença, susto ou desespero. No tocante às reações a perdas repentinas (não esperadas), as reações indicadas pelas respostas dos adolescentes foram: tristeza, ressentimento, dor, autocomiseração, desespero, resignação, desorientação, culpa por não ter podido evitar o ocorrido, raiva e revolta contra o destino. Já as reações descritas para as situações de perda esperada foram: raiva, isolamento e atitudes agressivas. Salientamos, conforme o estudo, que a reação depende em grande parte da proximidade com a pessoa e as circunstâncias da morte. Além disso, acreditamos que existam reações que podem estar presentes em qualquer tipo de morte (esperada ou não), tais como: tristeza e dor.

Após ter passado pela adolescência, o ser-humano entra na idade adulta, etapa na qual a morte vai além dos conceitos de universalidade e irreversibilidade. Nessa etapa, há um significado social, pois a morte de uma pessoa acarreta a mudança de papéis e relações na família. Sempre que uma pessoa idosa vem a falecer, todos os integrantes da família se movimentam uma etapa acima no sistema de gerações (BEE, 1997), além de aproximar o adulto de sua própria morte (BROWN, 2001).

No início da idade adulta os indivíduos concluem seus estudos e estão em meio a suas carreiras profissionais, seus casamentos e, provavelmente, possuem filhos. Estão ansiosos para viver tudo aquilo que planejaram e para o qual vinham se preparando. Assim, a morte pode ser encarada como motivo de frustração. Isso porque a morte nesta etapa faz com que o adulto se depare com sua finitude em um momento no qual os 
indivíduos lidam com questões do início da idade adulta, tais como os profissionais e familiares (PAPALIA; OLDS, 2000). Em estudo realizado com adultos jovens (20 a 30 anos), Zilberman (2002) investigou a concepção de morte no processo de individuação. Através deste estudo, foi possível concluir que existe evitação ou temor acerca do tema nos adultos jovens que se sentiam mais apegados ou dependentes de sua família. Além disso, constatou-se que a morte de alguém próximo poderia dificultar o processo de individuação que os adultos jovens vivenciam. Tal dado evidencia o impacto que a morte tem sobre o ser humano.

Por outro lado, as perdas da idade adulta (não somente a morte), podem trazer certo crescimento. Melo, Costa, Cardarelli e Moraes (2004) investigaram a elaboração de perda em mulheres adultas saudáveis, concluindo que diante das perdas houve possibilidade de fortalecimento, constituindo-se como uma forma de crescimento, o qual depende da passagem normal pelo luto. Diante destes dados, os quais evidenciam formas diferentes de enfrentamento diante da morte, podemos perceber 0 quanto tal acontecimento é gerador de diferentes reações, cada uma acompanhando uma etapa do processo de luto. Desde o temor e evitação do tema diante da ocorrência de morte até o fortalecimento advindo da situação, é notável o processo gradativo de assimilação da situação e a possibilidade de convivência com as perdas durante o desenvolvimento humano.

Passada a idade adulta, o ser humano se depara com a terceira idade, etapa do ciclo vital na qual há um número maior de perdas, colaborando para que o idoso pense mais sobre sua finitude (KOVÁCS, 2005). A perda de amigos e familiares, perda da sua ocupação, de parte de sua força física, redução do aparelho sensório e, em alguns casos, perda do funcionamento cerebral são comuns nesta idade (SILVA; CARVALHO; SANTOS; MENEZES, 2007). Em decorrência da terceira idade ser uma fase constituída por perdas, a morte nessa idade, conforme mencionam Lunardi e Lunardi Filho (1997) pode ser vista como natural e aceitável. Diante disso, é possível perceber que o tema morte é algo que acompanha freqüentemente os indivíduos de terceira idade. Bee (1997) nos diz que na velhice as pessoas tendem a pensar e falar mais sobre o assunto se comparadas a pessoas de qualquer outra faixa etária. Porém, tal fato não quer dizer que a temam menos do que pessoas de outras idades (ROSENBERG, 1992). De acordo com tais evidencias, pode-se pensar que a possibilidade eminente de morte que acompanha indivíduos nesta etapa do ciclo vital pode, em alguns casos, ser geradora de angustia. Segundo Rosenberg (1992, p. 70), "nosso medo da morte não caminha linearmente com nossa idade". Frumi e Celich (2006) realizaram uma pesquisa com cinco idosos acima de 60 anos com o objetivo de conhecer o significado do envelhecer e da morte para os idosos. Através do estudo, as autoras puderam perceber que os idosos encaram a morte como uma certeza, 
sendo que tal forma de encarar o assunto parece estar alicerçada em crenças e valores espirituais, que trazem para o idoso a confiança de uma vida após a morte. Sendo assim, é notável a importância de alguns aspectos, tais como a espiritualidade para uma vivência menos temerosa acerca da morte.

Silva, Carvalho, Santos e Menezes (2007) realizaram um estudo com o objetivo conhecer a vida do idoso após a morte de um amigo asilado. A pesquisa foi realizada com 15 idosos residentes em asilos permitindo concluir que diante da morte de um companheiro, os idosos se disseram chocados, descrentes, amedrontados, enfurecidos e tristes. Além disso, diversos sentimentos foram descritos, tais como sensação de vazio, falta do amigo, saudade, estarrecimento, tristeza, ansiedade, raiva, lembrança e solidão. $O$ estudo também evidenciou que a morte de um amigo pode representar aprendizado, uma vez que os idosos entrevistados citaram a dor, o sofrimento e a agonia como grandes momentos de aprendizado. Mais uma vez, podemos perceber a diversidade de reações diante da morte e que estas, independentemente da idade do indivíduo tendem a ter aspectos em comum, tais como a tristeza e o sofrimento.

Brown (2001) lembra que quanto mais tarde a morte ocorrer no ciclo de vida, menor será o estresse associado aos familiares e pessoas próximas, pois a morte numa idade avançada é encarada como algo natural. Porém a autora lembra que, mesmo que a morte de um idoso seja vista como parte integrante do ciclo de vida, isso não irá acontecer sem nenhum grau de estresse. Sem duvida, um fato como a morte, seja ela em qualquer fase do desenvolvimento humano, é vivenciado com tristeza e estresse. Tal fato pode ser explicado em parte pelo despreparo que nossa sociedade tem em lidar com o assunto, necessitando assim, preparo profissional para auxílio a quem necessite.

\section{Conclusões e Considerações Finais}




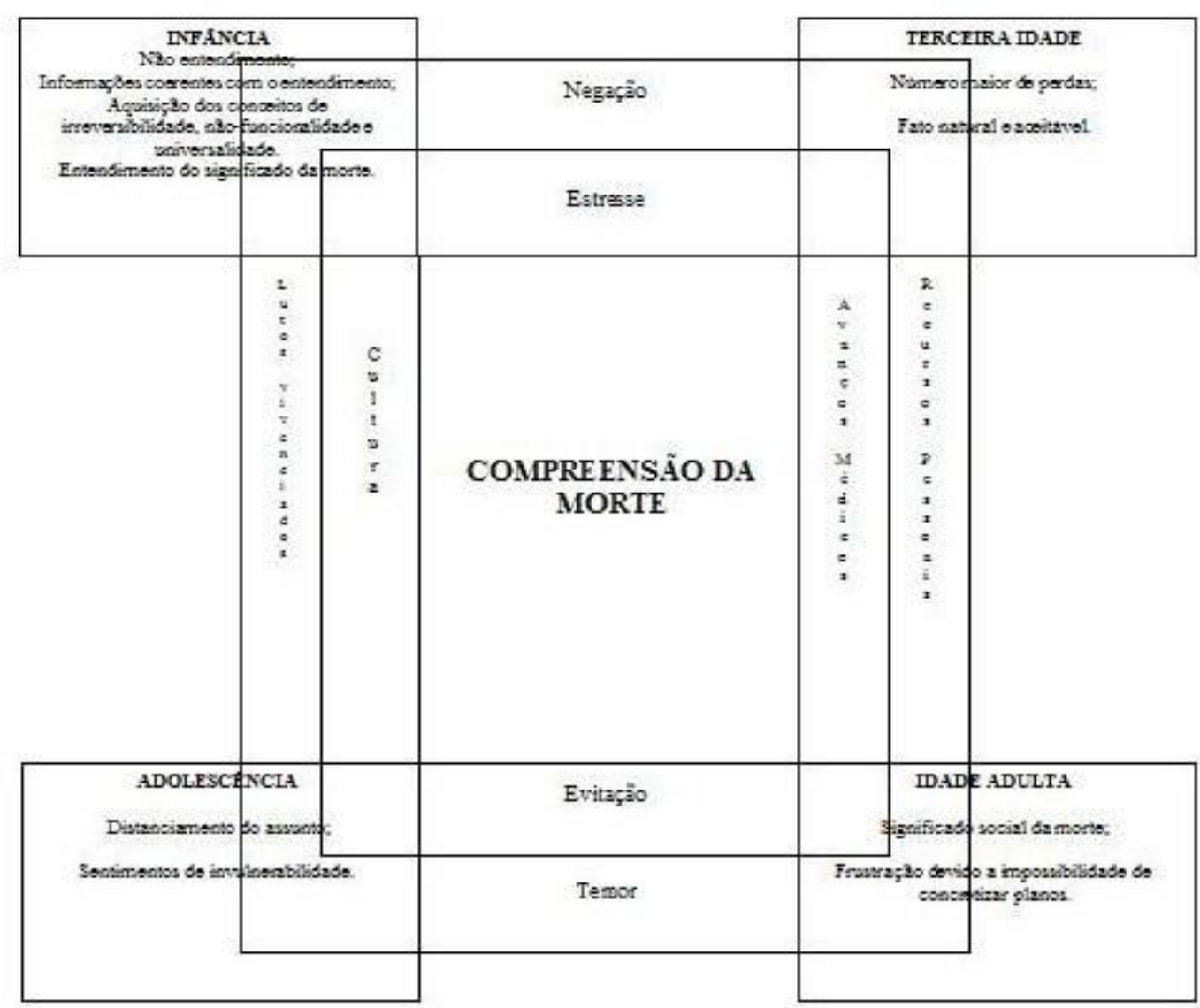

Com a realização deste estudo, buscou-se um entendimento geral da compreensão da morte ao longo do desenvolvimento humano. Através da leitura de diversos artigos e a busca por embasamento teórico em livros, foi desenvolvido um quadro explicativo, englobando as quatro etapas do desenvolvimento humano abordadas, bem como os possíveis fatores que exercem influência nesta compreensão.

Conclui-se que as crianças até certa idade não são capazes de compreender os aspectos centrais da morte, e a importância de disponibilizar informações verdadeiras e adequadas ao entendimento infantil. Informações verdadeiras no sentido de não ocultar da criança a ocorrência da morte de alguém próximo, mas sim tornar o fato compreensível à linguagem da criança. $\mathrm{Na}$ adolescência, evidenciamos que o assunto parece ser ignorado, uma vez que o jovem se considera novo demais para pensar nesse assunto, acreditando ser invulnerável. Na vida adulta a possibilidade da morte se concretiza como algo realmente possível de acontecer, tendo um significado social atribuído. Porém, é somente na velhice que a morte é encarada como natural. Sendo assim, acredita-se que é possível ver a compreensão que os indivíduos têm acerca da morte em um continuum, iniciando pelo desconhecimento infantil, passando pelo entendimento no final da infância, pela crença adolescente de que tal evento não venha a ocorrer, seguido da 
compreensão adulta da morte como uma possibilidade real, finalizando pela naturalidade com a qual o idoso tende a encarar o assunto.

Percebe-se também, a influência de diferentes variáveis sobre a compreensão que se tem da morte. Os níveis de negação, evitação, estresse e temor podem contribuir para o distanciamento do assunto, bem como os avanços médicos, os fatores culturais e os lutos vivenciados ao longo do desenvolvimento. Todas essas variáveis parecem estar envolvidas no modo como se encara este assunto. Diante disso, é notável a inter-relação de diversos fatores quanto à compreensão da morte. Neste ponto reside a necessidade de se estudar tal assunto, conhecendo cada vez mais o papel de cada um destes fatores. Dessa forma, acredita-se que os profissionais da área da saúde poderão estar preparados para tal situação, podendo oferecer o cuidado necessário nestas ocasiões.

Com relação aos estudos pesquisados para a composição deste artigo, é notável a predominância da abordagem qualitativa empregada na coleta de dados. Os participantes da pesquisa geralmente foram pacientes hospitalizados, familiares ou pessoas que haviam enfrentado situações de perda e luto. Dessa forma, acredita-se que para a realização de pesquisas com a temática da morte faz-se necessário o extremo cuidado e zelo para com os participantes. O assunto morte, como é possível concluir neste estudo, mobiliza diversos sentimentos e estes precisam de um apoio. Assim, sugere-se que sempre seja oferecido a estes participantes o acompanhamento necessário, especialmente, o acompanhamento psicológico.

Faz-se necessário que haja preparo constante para a atuação profissional. Tal preparo somente é obtido através de estudos e conhecimentos específicos sobre o tema. Sugerimos a possibilidade, haja vista a necessidade, de os cursos voltados à área de saúde oferecerem subsídios com relação ao tema. De acordo com Boemer, Veiga, Mendes, Valle (1991), há a necessidade de se educar os profissionais de saúde para o tema morte, uma vez que essa é uma constante em seu ambiente profissional.

\section{Referências Bibliográficas}

ALMEIDA, E. C. O psicólogo no hospital geral. Psicologia: Ciência e Profissão, Brasília, v. 20, n. 3, p. 24-27, set. 2000.

ALMEIDA, F. A. Lidando com a morte e o luto por meio do brincar: a criança com câncer no hospital. Boletim de Psicologia, São Paulo, v. 55, n. 123, p. 149-167, dez. 2005.

ARAUJ O, P. V. R; VIEIRA, M. J. As atitudes do homem frente à morte e o morrer. Revista Texto \& Contexto Enfermagem, Santa Catarina, v. 10, n. 3, p. 101-117, set-dez. 2001. 
ASSUMPÇÃO, E. V. D. Os que partem, os que ficam. Petrópolis: Editora Vozes, 2001.

BEE, H. O ciclo vital. Porto Alegre: Artes Médicas, 1997.

BELLATO, R; CARVALHO, E. C. O jogo existencial e a ritualização da morte. Revista Latino-Americana de Enfermagem, São Paulo, v. 13, n. 1, p. 99-104, jan-fev. 2005.

BOEMER, M. R; VEIGA, E. V; MENDES, M. M. R; VALLE, E. R. M. O tema da morte: uma proposta de educação. Revista Gaúcha de Enfermagem, Porto Alegre, v. 12, n. 1, p. 26-32, jan. 1991.

BROWN, F. H. O impacto da morte e da doença grave sobre o ciclo de vida familiar. In: Carter, B.; McGoldrick, M. As mudanças no ciclo de vida familiar: uma estrutura para a terapia familiar Porto Alegre: Artmed, 2001. p. 393-414.

COMBINATO, D. S; QUEIROZ, M. C. Morte: uma visão psicossocial. Estudos de Psicologia, Natal, v. 11, n. 002, p. 209-216, maio-ago. 2005.

COSTA, W. C. Morte e desenvolvimento humano. In: Py, Ligia. Finitude: uma proposta para reflexão e prática em gerontologia. Rio de Janeiro: NAU, 1999. p. 55-63.

DOMINGOS, B; MALUF, M. R. Experiências de perda e de luto em escolares de 13 a 18 anos. Psicologia: Reflexão e Crítica, Porto Alegre, v. 16, n. 3, p. 577-589, 2003.

ELKIND, D. All grown up and no place to go. Reading, MA: AddisonWesley, 1984.

FIDELIS, W. M. Z. A morte e o morrer nas representações sociais dos alunos de curso de ensino médio de enfermagem. 2001. $98 \mathrm{f}$. Tese (Mestrado em Enfermagem) - Escola de Enfermagem, Universidade de São Paulo.

FRANCO, M. H. P; MAZORRA, L. Criança e luto: vivências fantasmáticas diante da morte do genitor. Estudos de Psicologia, Campinas, v. 24, n. 4, p. 503-511, Out-Dez. 2007.

FRUMI, C; CELICH, K. L. S. O olhar do idoso frente ao envelhecimento e à morte. Revista Brasileira de Ciências do Envelhecimento Humano, Passo Fundo, p. 92-100, jul-dez. 2006.

GAUDERER, E. C. A criança, a morte e o luto. Jornal de Pediatria, Rio de Janeiro, v. 62, n.3, p. 82-90, mar. 1987.

GÜNTHER, I. A. Preocupações de adolescentes ou os jovens têm na cabeça mais do que bonés. Psicologia: Teoria e Prática, Brasília, v. 12, p. 61-69, jan-abr. 1996.

KOVÁCS, M. J. Educação para a morte. Psicologia Ciência e Profissão, Brasília, v. 25, n. 3, p. 484-497, set. 2005.

Notícia: Wilma da Costa Torres (1934-2004): pioneira da

Tanatologia no Brasil. Psicologia: Teoria e Pesquisa, Brasília, v. 20, n.

1, p. 95-96, Jan-Abr. 2004. 
Morte no processo de desenvolvimento humano: a criança e o adolescente diante da morte. In: M. J. Kovács. Morte e desenvolvimento humano. São Paulo: Caso do Psicólogo, 1992, p. 5889.

A questão da morte e a formação do psicólogo. 1989. 211 p. Tese (Doutorado em Psicologia) - Instituto de Psicologia, Universidade de São Paulo. São Paulo, SP.

KÜBLER-ROSS, E. Sobre a Morte e o Morrer. São Paulo: Martins Fontes, 2005.

LUNARDI, V. L. \& LUNARDI Filho, W. D. A morte do idoso: um fato natural e aceitável. Texto e Contexto Enfermagem, Florianópolis, v. 6, n. 2, p. 322-329, maio-ago. 1997.

MAZORRA, L. Luto na infância. Encontro, Santo André, v. 6, p. 23-28, jan-dez. 2001.

MELO, S. A; COSTA, A; CARDARELLI, G; Moraes, L. S. C. Elaboração de perdas por mulheres maduras saudáveis. Interação em Psicologia,Paraná, v. 8, n. 1, p. 129-140, jan-jun. 2004.

MELO, L. L; VALLE, E. R. M. "E a luz está se apagando...": vivências de uma criança com câncer em fase terminal. Revista Brasileira de Enfermagem, Brasília, v. 52, n. 4, p. 566-575, 1999.

NUNES, D. C; CARRARO, L; JOU, G. I; SPERB, T. M. As crianças e o conceito de morte. Revista Psicologia Reflexão e Crítica, Porto Alegre, v. 11, n. 3, p. 579-590, 1998.

OKAMOTO, M. R. Y. A morte que invade espaços: vivências de profissionais na instituição hospitalar. 2004. 219 p. Dissertação (Mestrado em Psicologia) - Instituto de Psicologia, Universidade de São Paulo. São Paulo, SP.

PAPALIA, D. E; OLDS, S. W. Desenvolvimento Humano. Porto Alegre: Artes Médicas, 2000.

PAZIN-FILHO, A. Morte: considerações para a prática médica. Revista Medicina, Ribeirão Preto, v. 38, n. 1, p. 20-25, jan-mar. 2005.

QUINTANA, A. M; ARPINI, D. M. A atitude diante da morte e seu efeito no profissional de saúde: uma lacuna da formação? Revista Psicologia Argumento, Paraná, v. 19, n. 30, p. 45-50, 2002.

Rodriguez, C. F. O que os jovens têm a dizer sobre a adolescência e o tema da morte? 2005. 258 p. Dissertação (Mestrado em Psicologia) Instituto de Psicologia, Universidade de São Paulo. São Paulo, SP.

Rodriguez, C. F; Kovács, M. J. Falando de morte com adolescente. Estudos e Pesquisas em Psicologia, Rio de Janeiro, v. 5, n. 1, p. 127-143, jan-jun. 2005.

ROSENBERG, R. L. Envelhecimento e morte. In: M. J. Kovács. Morte e Desenvolvimento Humano. São Paulo: Casa do Psicólogo, 1992. p. 5889. 
SILVA, C. A; CARVALHO L. S; SANTOS, A. C. P. O; MENEZES, M. R. Vivendo após a morte de amigos: história oral de idosos. Texto e Contexto Enfermagem, Florianópolis, v. 16, n. 1, p. 97-104, jan-mar. 2007.

SILVA, A. L. P. O acompanhamento psicológico a familiares de pacientes oncológicos terminais no cotidiano hospitalar. I nteração em Psicologia, Paraná, v. 7, n. 1, p. 27-35, jan-jun. 2003.

SOUZA, L. G. A; BOEMEr, M. O cuidar em situação de morte: algumas reflexões. Revista Medicina, Ribeirão Preto, v. 38, n. 1, p. 49-54, janmar. 2005.

TORRES, W. C. O conceito de morte em crianças portadoras de doenças crônicas. Psicologia: Teoria e Pesquisa, Brasília, v. 18, n. 2, p. 221-229, maio-ago. 2002.

TONETTO, L. M; RECH, T. Lidar com a terminalidade: um desafio para o psicólogo. Revista Psico, Porto Alegre, v. 32, n. 1, p. 131-145, jan-jun. 2001.

ZAVASCHI, M. L. S; SATLER, F; POESTER, D; VARGAS, C. F; PIAZENSKI, R; ROHDE, L. A. P; EIZIRIK, C. L. Associação entre trauma por perda na infância e depressão na vida adulta. Revista Brasileira de Psiquiatria, São Paulo, v. 24, n. 4, p. 189-195, Out. 2002.

ZILBERMAN, A. B. A concepção de morte em adultos jovens no processo de individuação. 2002. 82 p. Dissertação (Mestrado em Psicologia) - Faculdade de Psicologia, Pontifícia Universidade Católica do Rio Grande do Sul. Porto Alegre. Rio Grande do Sul, RS.

\author{
Endereço para correspondência \\ Jean Von Hohendorff \\ Faculdades Integradas de Taquara, Curso de Psicologia, Av. Oscar Martins Rangel, 4500 \\ (RS 115), Taquara, RS, Brasil. CEP: 95600-000 \\ Endereço eletrônico: jhohendorff@gmail.com \\ Wilson Vieira Melo \\ WP Centro de Psicoterapia Cognitivo-Comportamental, Rua Santa Cecília, 1556, Porto \\ Alegre, RS, Brasil. CEP: 95600-000 \\ Endereço eletrônico: melo@terapiascognitivas.com.br \\ Recebido em: 24/10/2008 \\ Aceito para publicação em: 04/03/2009 \\ Editor responsável: Adriana Benevides Soares
}

Notas

* Doutorando em Psicologia na Universidade Federal do Rio Grande do Sul-UFRGS 